\title{
Comparison of Two Multidisciplinary Optimization Strategies for Launch-Vehicle Design
}

\author{
R. D. Braun, ${ }^{*}$ R. W. Powell, ${ }^{*}$ R. A. Lepsch, ${ }^{*}$ and D. O. Stanley* \\ NASA Langley Research Center, Hampton. Virginia 23681 \\ and \\ I. M. Kroo ${ }^{\dagger}$ \\ Stanford University, Stanford, California 94305
}

\begin{abstract}
The investigation focuses on development of a rapid multidisciplinary analysis and optimization capability for launch-vehicle design. Two multidisciplinary optimization strategies in which the analyses are integrated in different manners are implemented and evaluated for solution of a single-stage-to-orbit launch-vehicle design problem. Weights and sizing, propulsion, and trajectory issues are directly addressed in each optimization process. Additionally, the need to maintain a consistent vehicle model across the disciplines is discussed. Both solution strategies were shown to obtain similar solutions from two different starting points. These solutions suggests that a dual-fuel, single-stage-to-orbit vehicle with a dry weight of approximately $1.927 \times 10^{5} \mathrm{lb}$, gross liftoff weight of $2.165 \times 10^{6} \mathrm{lb}$, and length of $181 \mathrm{ft}$ is attainable. A comparison of the two approaches demonstrates that treatment of disciplinary coupling has a direct effect on optimization convergence and the required computational effort. In comparison with the first solution strategy, which is of the general form typically used within the launch vehicle design community at present, the second optimization approach is shown to be 3-4 times more computationally efficient.
\end{abstract}

\begin{tabular}{ll} 
& \multicolumn{1}{c}{ Nomenclature } \\
$A_{r}$ & $=$ nozzle exit area, $\mathrm{ft}^{2}$ \\
$c$ & $=$ nonlinear constraint vector \\
$F_{z}$ & $=$ wing normal force, $\mathrm{lb}$ \\
GLOW & $=$ vehicle gross liftoff weight, $\mathrm{lb}$ \\
$I_{\mathrm{sp}}$ & $=$ specific impulse, $\mathrm{s}$ \\
$J$ & $=$ objective function \\
$\mathrm{MR}$ & $=$ mass ratio \\
$S_{\text {icf }}$ & $=$ reference aerodynamic surface area, $\mathrm{ft}^{2}$ \\
$T$ & $=$ thrust, $\mathrm{b}$ \\
$T / W$ & $=$ thrust-to-weight ratio \\
$x$ & $=$ design variable vector \\
$\alpha$ & $=$ angle of attack, deg \\
$\Sigma$ & $=$ discipline compatibility tolerance
\end{tabular}

Subscripts

c $\quad=$ computed

vac $=$ vacuum

sl $\quad=$ sea level

\section{Introduction}

$\mathbf{F}_{\mathrm{t}}$ OR several years, various Earth-to-orbit transportation options have been examined with the goal of reducing operating costs relative to the current U.S. launch feet. ${ }^{1,2}$ Many of these solutions have focused on fully reusable systems employing various levels of advanced technology. ${ }^{3}$ Although a wide range of options have been examined, including single- and two-stage systems using rocket and/or air-breathing propulsion, current emphasis has been placed on single-stage-to-orbit, rocket-powered vehicles. ${ }^{3-5}$ The design of

Received Oct. 17, 1994; revision received Feb. 13, 1995; accepted for publication Feb. 15, 1995. Copyright (C) 1995 by the American Institute of Aeronautics and Astronautics, Inc. No copyright is asserted in the United States under Title 17. U.S. Code. The U.S. Government has a royalty-free license to exercise all rights under the copyright claimed herein for Governmental purposes. All other rights are reserved by the copyright owner.

*Aerospace Engineer, Space Systems and Concepts Division. Member AIAA

${ }^{\dagger}$ Associate Professor, Department of Aeronautics and Astronautics. Member AIAA such a vehicle is a multidisciplinary process in which aerodynamics, propulsion, weights and sizing, structures, performance, heating, operations, and cost must be addressed ${ }^{5}$ Although it is imperative that each of these disciplines be addressed at the conceptual design level. it is equally vital to be able to perform this multidisciplinary analysis and optimization rapidly so that the numerous design options may be evaluated and understood.

The present investigation focuses on development of a rapid multidisciplinary analysis capability for launch-vehicle design. The specific application chosen is that of a dual-fuel, single-stage-to-orbit launch vehicle. Two multidisciplinary optimization strategies are implemented and evaluated for the solution of this problem, and differences among the approaches are highlighted. Weights and sizing, propulsion, and trajectory issues are directly addressed in the optimization processes. Additionally, the need to maintain a consistent vehicle model across the disciplines is discussed.

Problem Definition and Disciplinary Analyses

In this analysis, design of a single-stage-to-orbit launch-vehicle includes specification of the ascent trajectory through the initial launch azimuth, time of flight, and pitch-angle history. Determination of the appropriate component weights and sizes is performed, and the vehicle dry weight (defined as the vehicle weight without payload, propellant, fluids, or crew) is selected as the minimization variable. Table 1 summarizes the design variables and constraints that characterize this optimization problcm. Propulsionsystem characteristics to be optimized include the liftoff thrust-toweight ratio, two nozzle area ratios, and two fuel-to-oxidizer mixture ratios. In this analysis, a dual-position nozzle is used to provide the performance benefit of a smaller nozzle exit area at liftoff (to maximize sea-level thrust) while allowing for a larger expansion at high altitudes (to maximize vacuum thrust). Two mixture ratios require specification because the vehicle is operated in different propulsive modes. Beginning with liftoff, hydrogen and kerosene are both burned as fucl, but during a later portion of the ascent, hydrogen becomes the sole fuel. Such a dual-fuel strategy has been shown to provide significant dry-weight reductions." As listed in Table 1, the transition time from mode 1 to mode 2 propulsion and the extension of the dual-position nozz re also optimally determined.

The vehicle is sized 1 iver and return a 25,000 -lh payload to the Space Station follo aunch from the Eastern Test Range at 


\section{Comparison of Two Multidisciplinary Optimization Strategies for Launch-Vehicle Design R. D. Braun, R. W. Powell, R. A. Lepsch, D. 0. Stanley, I. M. Kroo}

Reprinted from

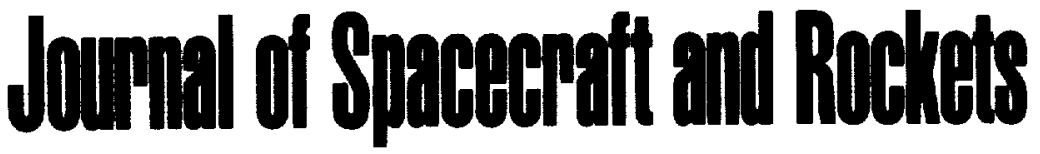

Volume 32, Number 3, Pages 404-410

\section{JAIAA:}


Table 1 Single-stage-to-orbit launch-vehicle design characteristics for iterative-loop and sequential compatibility-constraint methods (objective function $=$ dry weight)

\begin{tabular}{|c|c|c|c|c|c|}
\hline \multirow[b]{2}{*}{ Iteration } & \multirow[b]{2}{*}{ Design variables } & \multirow[b]{2}{*}{ Min. } & \multirow[b]{2}{*}{ Max. } & \multicolumn{2}{|c|}{ Initial value } \\
\hline & & & & 1 & 2 \\
\hline 1 & GLOW: ib & $1.5 \mathrm{e} 6$ & $3.0 \mathrm{e} 6$ & $2.0 \mathrm{e} 6$ & $2.5 \mathrm{e} 6$ \\
\hline 2 & $S_{\text {ref }}^{\mathrm{a}} \mathrm{ft}^{2}$ & $2.5 \mathrm{e} 3$ & $5.0 \mathrm{e} 3$ & $4.0 \mathrm{e} 3$ & $3.0 \mathrm{e} 3$ \\
\hline 3 & Landed weight, ${ }^{a}$ lb & $1.0 \mathrm{e} 5$ & $3.0 \mathrm{e} 5$ & $2.2 \mathrm{e} 5$ & $2.0 \mathrm{e} 5$ \\
\hline 4 & Base diameter, ${ }^{a t}$ & 20.0 & 40.0 & 30.0 & 25.0 \\
\hline 5 & Launch azimuth, deg & 20.0 & 60.0 & 45.0 & 40.0 \\
\hline 6 & Vehicle liftoff $T / W$ & 1.2 & 1.5 & 1.3 & 1.4 \\
\hline 7 & $\% \mathrm{LH}_{2}$, mode 1 & 3.0 & 15.0 & 6.0 & 4.0 \\
\hline 8 & Mode 2 mixture ratio & 3.0 & 15.0 & 7.5 & 9.0 \\
\hline 9 & Nozzle area ratio, retracted & 40.0 & 100.0 & 60.0 & 50.0 \\
\hline 10 & Nozzle area ratio, extended & 60.0 & 200.0 & 120.0 & 140.0 \\
\hline 11 & Propulsion mode transition Mach number & 2.0 & 20.0 & 6.0 & 5.0 \\
\hline 12 & Nozzle transition Mach number & 0.0 & 8.0 & 1.0 & 2.0 \\
\hline 13 & $F_{Z}$ boundary duration, $\mathrm{s}$ & 20.0 & 150.0 & 80.0 & 100.0 \\
\hline 14 & Total trajectory time, s & 300.0 & 500.0 & 380.0 & 400.0 \\
\hline $15-40$ & Set of pitch angle, ${ }^{b} \mathrm{deg}$ & -1120.0 & 0.0 & Quadratic & Linear \\
\hline Iteration & Constraints & \multicolumn{2}{|c|}{ Min. } & \multicolumn{2}{|c|}{$\operatorname{Max}$} \\
\hline 1 & Terminal altitude, $\mathrm{ft}$ & \multicolumn{2}{|c|}{$3.03505 \mathrm{e} 5$} & \multicolumn{2}{|c|}{$3.04105 \mathrm{e} 5$} \\
\hline 2 & Terminal velocity, ft/s & \multicolumn{2}{|c|}{$2.5843 \mathrm{e} 4$} & \multicolumn{2}{|c|}{$2.5845 \mathrm{e} 4$} \\
\hline 3 & Terminal flight-path angle, deg & \multicolumn{2}{|c|}{$-1.0 \mathrm{e}-03$} & \multicolumn{2}{|c|}{$1.0 \mathrm{e}-03$} \\
\hline 4 & Terminal inclination, deg & \multicolumn{2}{|c|}{51.5} & \multicolumn{2}{|c|}{51.7} \\
\hline 5 & Dynamic pressure, $1 \mathrm{~b} / \mathrm{ft}^{2}$ & \multicolumn{2}{|c|}{0.0} & \multicolumn{2}{|c|}{1000.0} \\
\hline 6 & $F_{Z}$ magnitude, lb & \multicolumn{2}{|c|}{0.0} & \multicolumn{2}{|c|}{$2.5 \times$ landed weight } \\
\hline 7 & Nozzle extension ratio & \multicolumn{2}{|c|}{0.0} & \multicolumn{2}{|c|}{2.0} \\
\hline 8 & $\alpha, \operatorname{deg}$ & \multicolumn{2}{|c|}{-15.0} & \multicolumn{2}{|c|}{15.0} \\
\hline 9 & Pitch rate, deg/s & \multicolumn{2}{|c|}{-5.0} & \multicolumn{2}{|c|}{-5.0} \\
\hline 10 & GLOW compatibility." lb & \multicolumn{2}{|c|}{$-1.0 \mathrm{e} 4$} & \multicolumn{2}{|c|}{$1.0 \mathrm{e} 4$} \\
\hline 11 & $S_{\text {ref }}$ compatibility ${ }^{\mathrm{c}} \mathrm{ft}^{2}$ & \multicolumn{2}{|c|}{-5.0} & \multicolumn{2}{|c|}{5.0} \\
\hline 12 & Landed weight compatibility. lb & \multicolumn{2}{|c|}{$-1.0 \mathrm{e} 2$} & \multicolumn{2}{|c|}{$1.0 \mathrm{e} 2$} \\
\hline 13 & Base diameter compatibility, ${ }^{\mathrm{c}} \mathrm{ft}$ & & & & \\
\hline
\end{tabular}

${ }^{2}$ Not treated as a design variable in the iterative-loop approach (Fig 4).

"In this system, 0 deg is vertical; \pm 90 deg is horizontal.

'Not treated as a constraint in the iterative-loop approach (Fig. 4).

the Kennedy Space Center. For this analysis, the Space Station is assumed to be in a 220 -n-mile-altitude, circular orbit with a 51.6deg inclination. The single-stage-to-orbit vehicle is initially inserted into an orbit with an altitude of $50 \times 100 \mathrm{n}$ mile with the correct inclination. Onboard propellant is then used to transfer to and circularize at $220 \mathrm{n}$ mile. This maneuver is accomplished by two engines, each with a vacuum thrust of $6000 \mathrm{lb}$ and vacuum $I_{\mathrm{sp}}$ of $462.2 \mathrm{~s}$.

Terminal constraints on altitude, velocity, flight-path angle, and inclination are enforced, as are maximum in-flight normal-force, angle-of-attack, and dynamic-pressure limits (see Table 1). Each of these in-flight constraints is included to reflect an analysis restriction derived outside the scope of the present investigation. For example, a limit on the allowable normal force is included such that the ascent wing loading does not exceed structural limits $(2.5$ times the landed weight) derived in an entry sizing analysis. Furthermore, an angle-of-attack constraint is included such that the validity range of the aerodynamic analysis is not exceeded. Sizing restrictions require that a limit be placed on the allowable extension of the dual-position nozzle such that a maximum 2 -to- 1 increase in exit area results. A pitch-rate limit of $5 \mathrm{deg} / \mathrm{s}$ (to reflect control issues that are not modeled) and a maximum $3 g$ acceleration limit are also imposed. Note that since the maximum acceleration is enforced within the trajectory analysis by engine throttling, not by the optimizer, a maximum acceleration limit is not listed in Table 1 .

To simplify the analysis so that the problem is tractable, several design disciplines were decoupled from the present analysis. An existing vehicle geometry, aerodynamics database, and internal packaging analysis were used. ${ }^{7}$ Data from aerodynamics, structures, heating, and other subsystems were fixed or scaled appropriately. Of major significance for the current optimization study are the disciplines of propulsion, performance, and weights and sizing, each of which is modeled here.

For this analysis, propulsion-system parametrics supplied by Pratt and Whitney, based on modification of the proposed Russian RD-701 dual-fuel engine, were used. ${ }^{8}$ After a regression analysis,

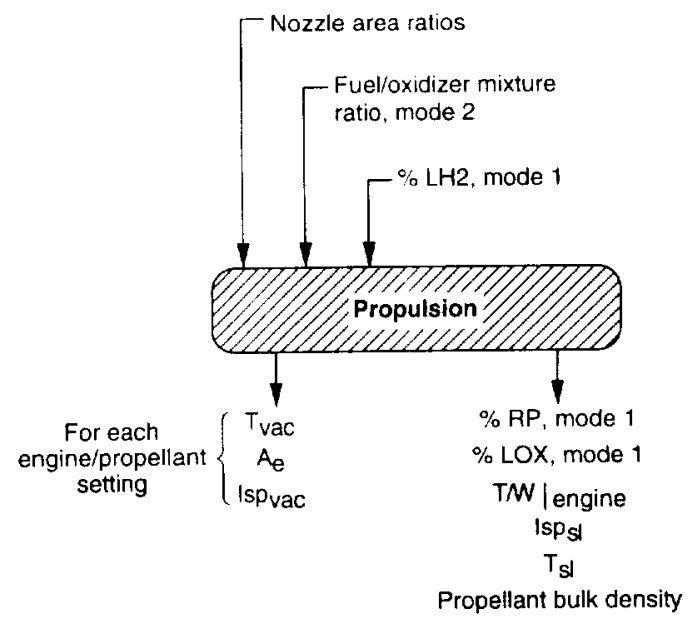

Fig. 1 Propulsion analysis inputs and outputs.

this parametric data set is used as shown in Fig. 1. Given the nozzle area ratios and fuel-to-oxidizer mixture ratios, numerous engine parameters are computed. These parameters include the sea-level engine thrust-to-weight ratio, sea-level specific impulse, and propellant bulk density (required inputs to the weights and sizing analysis), as well as the vacuum thrust, vacuum specific impulse, and nozzle exit area (required trajectory inputs).

In this investigation, the three-degree-of-freedom equations of motion were numerically integrated with use of the Program to Optimize Simulated Trajectories (POST). ${ }^{9}$ The vehicle is treated as a point mass; Earth rotation and oblateness are modeled, and the 1976 standard atmosphere is used. As shown in Fig. 2, the required set of POST inputs includes vehicle parameters (e.g., gross liftoff weight, vehicle thrust-to-weight ratio at liftoff, aerodynamic coefficients, 


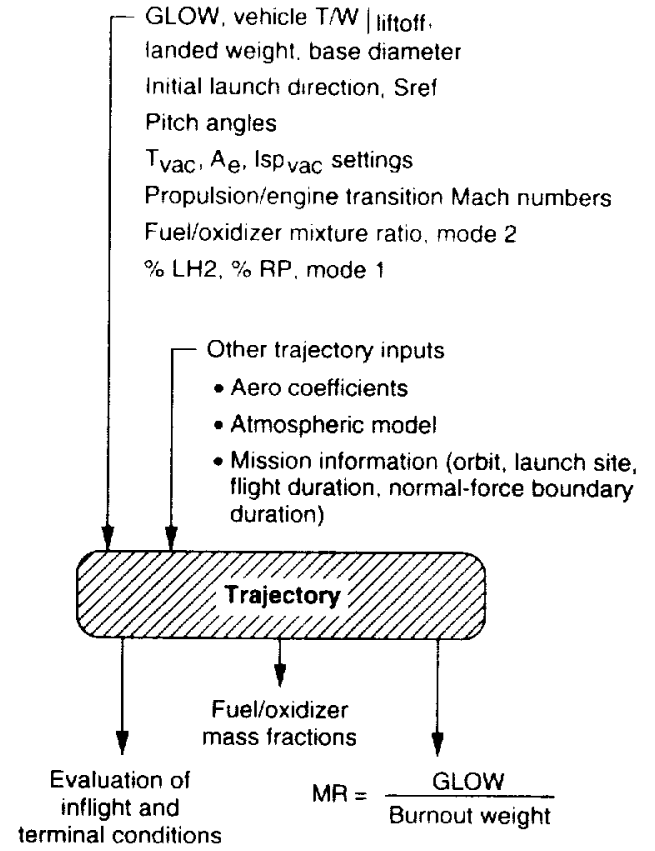

Fig. 2 Trajectory analysis inputs and outputs.

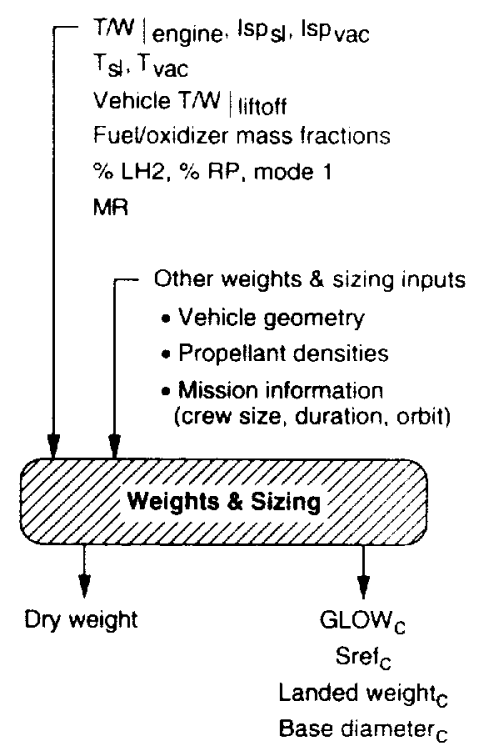

Fig. 3 Weight and sizing analysis inputs and outputs.

and reference area) as well as trajectory parameters (pitch-angle history, launch azimuth, and propulsion-system transition Mach numbers) and propulsion parameters (propellant mixture ratios and engine performance characteristics). POST is used to evaluate the in-flight and terminal constraints and to compute the vehicle mass ratio and required propellant fractions (weights and sizing inputs).

For a given mass ratio, the configuration sizing program (CONSIZ), developed at NASA Langley Research Center, is used to size the vehicle and determine the dry weight. Within CONSIZ, the vehicle is modeled as a collection of components representing structure, subsystem, and propulsion elements. Specific weight-estimating relationships for each component are considered part of the program input. This provides user flexibility in both vehicle modeling and analysis depth. As shown in Fig. 3, the liftoff thrust-to-weight ratio, mass ratio, and propellant fractions, as well as several other propulsion system parameters, are required inputs to CONSIZ. Note that, with the exception of the liftoff thrust-to-weight ratio, all of these inputs are computed by one of the other two disciplinary analyses. In addition to dry weight, CONSIZ computes the gross liftoff weight, reference aerodynamic surface area, base diameter, and landed weight (each of which is a required trajectory input).
From Figs. 1-3, it is clear that solution of this problem requires an iterative approach because the trajectory and weights and sizing analyses each require inputs that are computed by another discipline. For example, one must ensure that the reference aerodynamic surface area resulting from the vehicle sizing process ( $S_{\text {ref, }}$ of Fig. 3) is the same as the reference aerodynamic surface area used to compute the aerodynamic forces and evaluate the flight path ( $S_{\mathrm{rct}}$ of Fig. 2). Consistency must also be maintained in regard to the gross liftoff weight, base diameter, landed weight (which effects the ascent normal-force limit), mass ratio, propellant fractions, and each of the propulsion discipline outputs. Furthermore, the liftoff thrustto-weight and fucl-to-oxidizer mixture ratios must be treated in a similar fashion, since these parameters are input to more than one discipline.

\section{Optimization Approaches}

The disciplinary analysis tools used in this study were originally created as independent programs, each operated by a disciplinary expert. To obtain a feasible point design vehicle, a design team was required to iterate manually among these disciplinary analyses. In many cases, "optimization" was performed through trade studies in which the parameters were varied one at a time. Significant improvement over this one-variable-at-a-time, parametric approach has been achieved using response-surface methods (RSMs). ${ }^{5-7}$ In this multidisciplinary strategy, feasible designs are computed at numerous statistically selected points in the design space, and a surface is fitted to these points. Optimization is then performed on this approximate representation of the design space. As another means of comparison, the launch-vehicle design problem discussed in the present investigation is solved using system sensitivity analysis (SSA) in Ref. 10. The present results are compared with those obtained with SSA in that reference.

The present investigation considers two solution approaches in which the previously independent disciplinary tools are integrated. These two optimization strategies will be referred to as 1) the iterative-loop approach and 2) the sequential compatibilityconstraint method. For this particular problem and set of analysis tools, these integrated approaches provide several advantages over the use of RSMs. These advantages include the following: 1) optimization over the actual design space as opposed to the use of an approximate surface, 2) no human interface time requirement to negotiate between disciplinary programs, and 3 ) the guarantee of a consistent multidisciplinary vehicle model at the solution. The primary disadvantage to the use of either of these integrated strategies is the up-front time required to integrate the disciplinary tools in a general manner. For example, prior to integration with the other disciplinary analyses, several modifications to CONSIZ were required so that accurate derivative information could be obtained in a rapid fashion. The lack of sensitivity information at design points other than the optimum is another drawback of the integrated approaches.

\section{Results and Discussion}

For each optimization method, solution of the single-stage-toorbit problem is attempted from the two starting points listed in Table 1. The first starting point may be viewed as a good initial guess, whereas starting point 2 is quite poor. For each solution strategy, optimization was performed with the sequential quadratic programming algorithm NPSOL." NPSOL uses a quasi-Newton method to approximate the Hessian of the Lagrangian. This algorithm is known to converge to local minimum for problems that are scaled properly and are twice continuously differentiable.

Prior to optimization (with each solution strategy), the problem was scaled so that the design variables, constraints, and objective function were all of order one. However, in this investigation, no attempt was made to provide a twice continuously differentiable model. Sources that contribute to this nonsmoothness include the ascent pitch profile, which is modeled by discrete control points connected by linear segments, and the atmospheric properties (1976 standard model). Hence, in this analysis, NPSOL is not expected to achieve a tightly converged, optimal solution, but rather to identify near-optimal solutions useful at the conceptual design level. All 
computations were performed on a Silicon Graphics Challenge L machine outfitted with $100-\mathrm{MHz}$ processors; CPU times quoted are based on use of this computational platform.

\section{Iterative-Loop Method}

The first approach toward an integrated multidisciplinary solution strategy is depicted in Fig. 4. Here, an iterative loop is set up between the trajectory and weights and the sizing disciplines; values of GLOW, $S_{\text {ref }}$, the base diameter, and the landed weight are used as loop convergence criteria. This type of strategy has been employed by other investigators ${ }^{12-14}$ for solution of launch-vehicle design problems. Indeed, as an integrated design tool, this method should be viewed as the current way of doing business within the space transportation community. The popularity of the approach is largely a result of the tool-integration simplicity. Using the terminology of Ref. 15, this formulation may be referred to as "multidisciplinary feasible" in that, for each set of design variables, the looped analyses return a design candidate that is consistent across disciplinary boundaries.

Using this iterative-loop formulation, the single-stage-to-orbit launch-vehicle design problem was solved from each of the two starting points listed in Table 1. Each of these solutions is summarized in Table 2. Although the solution that began at point 2 required 10 extra iterations, it is evident that both optimization processes converged to approximately the same point. At the solution, the vehicle liftoff thrust-to-weight ratio is the only variable on its bound (a liftoff $T / W<1.2$ is not allowed for abort reasons).

The vehicle's ascent profile is summarized in Figs. 5 and 6 for the solution that began at starting point 1 . Flight begins with a $400-\mathrm{ft}$ vertical rise to clear the launch facility. This is followed by a maximum-pitch-rate segment in which the vehicle is trying to attain a maximum-lift orientation. The pitch rate is limited to $5 \mathrm{deg} / \mathrm{s}$ to reflect control issues that are not modeled in this analysis. As shown in Fig. 6, during this segment of flight, the angle of attack $(\alpha)$ increases until it reaches the allowed maximum of $15 \mathrm{deg}$. Flying at this $\alpha$, the normal force builds until a limit load is reached. The vehicle rides this normal-force boundary through peak dynamic-pressure, which for the optimum flight path is about 830 psf. Hence, the dynamic pressure limit of $1000 \mathrm{psf}$ is not active. During this phase of flight, at approximately $18 \mathrm{kft}$, the back-pressure losses are low enough that the dual-position nozzle is extended to gain propulsive efficiency. After the nozzle extension is completed, the vehicle acceleration initially decreases as a result of flight through the transonic regime. At

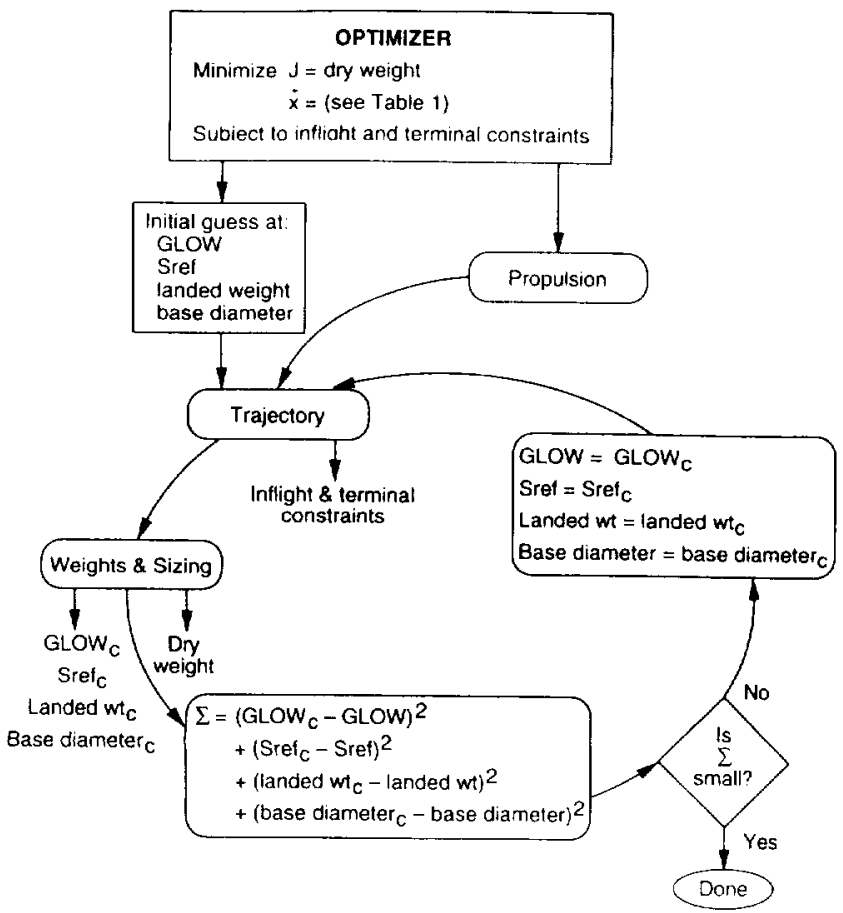

Fig. 4 Iterative-loop solution strategy.
Table 2 Single-stage-to-orbit launch-vehicle design solution: summary for iterative-loop method

\begin{tabular}{lcc}
\hline \hline & \multicolumn{2}{c}{ From initial point } \\
\cline { 2 - 3 } & & 2 \\
\hline Looped variables & & \\
GLOW, lb & $2.1655 \mathrm{e}+06$ & $2.1678 \mathrm{e}+06$ \\
$S_{\text {ref, }} \mathrm{ft}^{2}$ & $4.0476 \mathrm{e}+03$ & $4.0498 \mathrm{e}+03$ \\
Landed weight, $\mathrm{lb}$ & $2.2100 \mathrm{e}+05$ & $2.2102 \mathrm{e}+05$ \\
Base diameter, $\mathrm{ft}$ & 27.916 & 27.906 \\
Design variables & & \\
Launch azimuth, deg & 42.458 & 42.458 \\
Vehicle liftoff $T / W$ & 1.2000 & 1.2000 \\
\% LH ${ }_{2}$, mode 1 & 6.2587 & 6.2190 \\
Mode 2 mixture ratio & 6.9986 & 6.9985 \\
Nozzle area ratio, retracted & 59.675 & 59.687 \\
Nozzle area ratio, extended & 119.36 & 119.30 \\
Propulsion mode transition & & \\
$\quad$ Mach number & 7.31 .37 & 7.3608 \\
Nozzle transition Mach number & 0.8904 & 0.9011 \\
$F_{Z}$ boundary duration, $\mathrm{s}$ & 55.155 & 60.248 \\
Total trajectory time, s & 377.61 & 377.62 \\
Performance & & \\
Final objective (dry weight, lb) & $1.92676 \mathrm{e}+05$ & $1.92695 \mathrm{e}+05$ \\
Number of iterations & 65 & 75 \\
Number of analysis calls & 10482 & 13101 \\
CPU time. h & 13.3 & 18.2 \\
\hline \hline
\end{tabular}

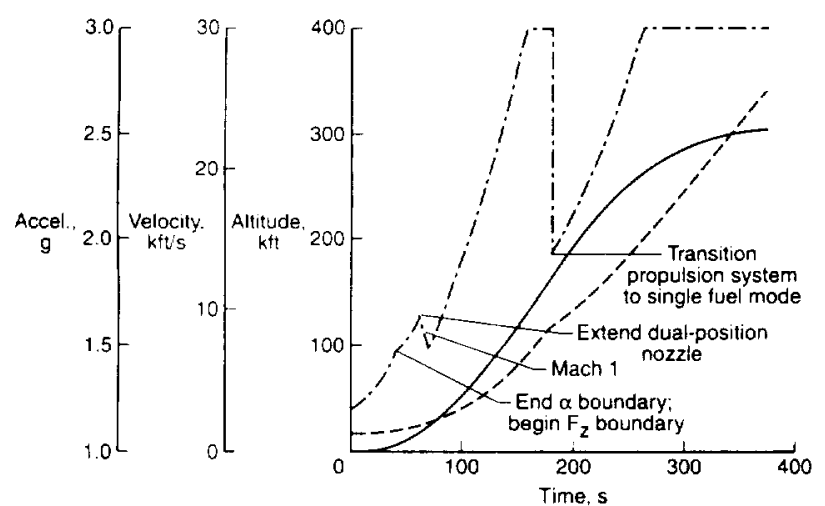

Fig. 5 Optimal ascent altitude, velocity, and acceleration profiles obtained through application of the iterative-loop method: - , altitude; --- , velocity; - - - acceleration.

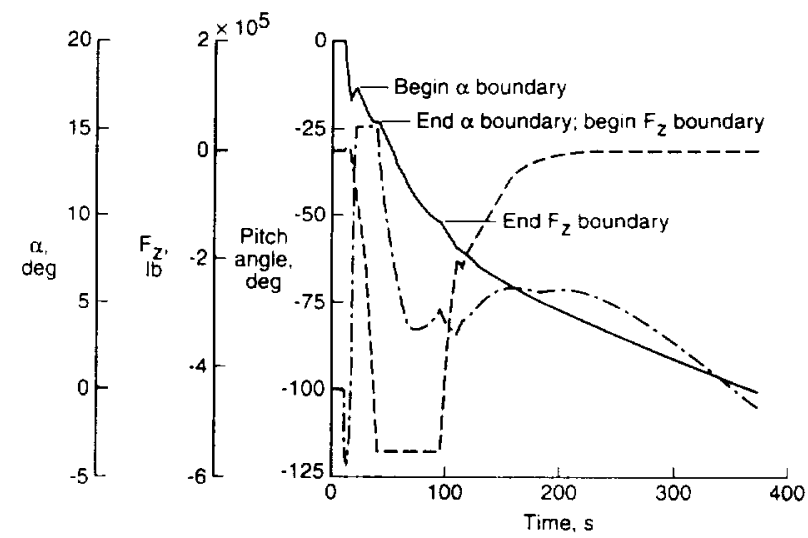

Fig. 6 Optimal ascent pitch-angle, normal-force, and angle-of-attack profiles obtained through application of the iterative-loop method: - pitch angle;,$--- F_{Z} ;---, \alpha$.

about $47 \mathrm{kft}$, as the dynamic pressure decreases, the vehicle comes off the normal-force boundary but continues to accelerate towards $3 g$. The vehicle reaches $3 g$ while in the dual-fuel propulsive mode and throttles down the engines to maintain this level of acceleration. In this analysis, transition of all seven engines from a dual-fuel to single-fuel mode is performed instantaneously. This results in the large decrease in acceleration shown in Fig. 5 at roughly $180 \mathrm{~s}$ and an altitude of about $164 \mathrm{kft}$. (A slightly better dry weight would 


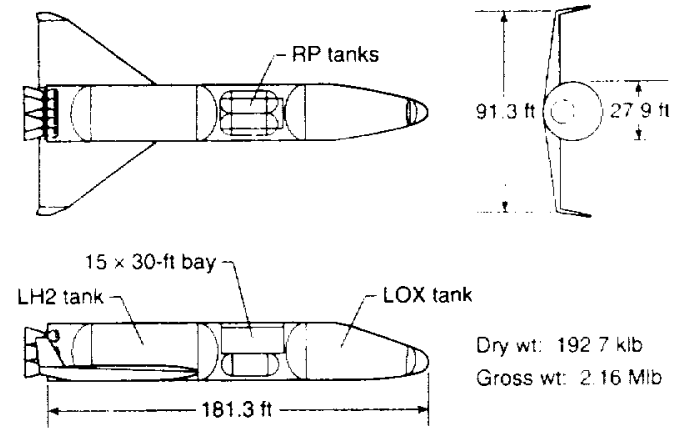

Fig. 7 Optimal single-stage-to-orbit vehicle description obtained through application of the iterative-loop method.

a)
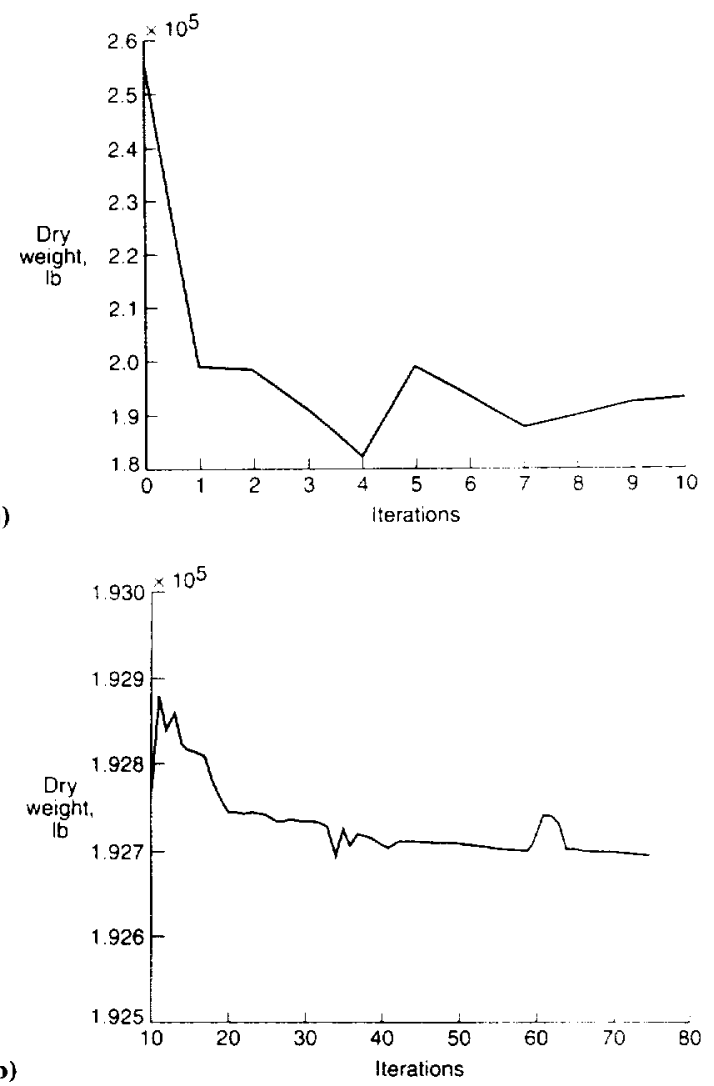

b)

Fig. 8 Objective function (dry weight) convergence history for the iterative-loop solution: a) first 10 iterations and b) final 65 iterations (note scale change).

result if the engines were allowed to transition sequentially.) Operating in a single-fuel mode $\left(\mathrm{LH}_{2}\right)$, the vehicle accelerates back to $3 \mathrm{~g}$ and holds this acceleration until reaching orbit.

Both solutions suggest that a vehicle dry weight of approximately $192,700 \mathrm{lb}$ is attainable for a 28 - $\mathrm{ft}$-diam configuration $(181.3 \mathrm{ft}$ in length) with a gross liftoff weight of about $2.165 \times 10^{6} \mathrm{Ib}$ and a wing area of $4050 \mathrm{ft}^{2}$. Such a configuration is sketched in Fig. 7.

Figures 8 and 9 show the objective function and nonlinear constraint convergence histories beginning from the more demanding starting point. Note 1) the dry-weight scale change in going from Fig. $8 \mathrm{a}$ to Fig. $8 \mathrm{~b}$ and 2 ) the semilog format of Fig. 9, which depicts the norm of the constraint violations. From these figures, it is clear that after approximately 10 iterations the objective function achieves a near-optimum value, whereas the constraints are not satisfied to the prescribed level (indicated by the nonlinear feasibility tolerance). These optimization characteristics are a result of the rather flat design space in which the most difficult task is to find a consistent vehicle model that satisfies all of the constraints. Lack of model smoothness is also evident in Fig. 9 and may have hampered the convergence rate. Note that at about iteration 60 , after the optimizer has formed a good model of the design space, a large

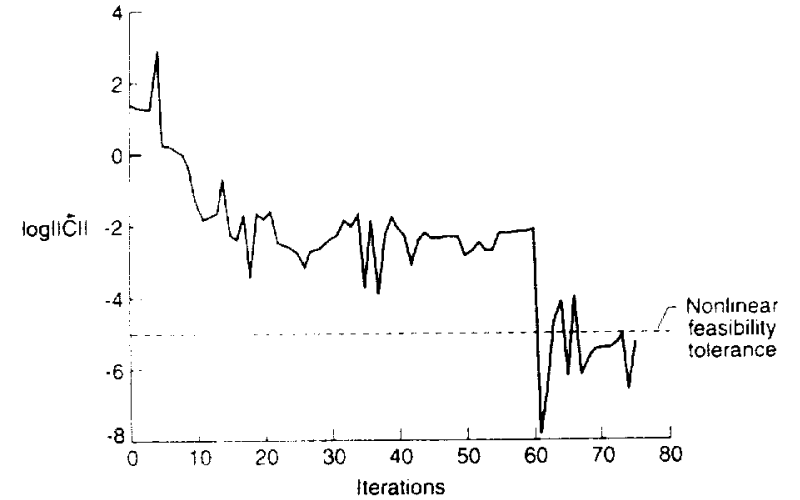

Fig. 9 Nonlinear-constraint convergence history for the iterative-loop solution.

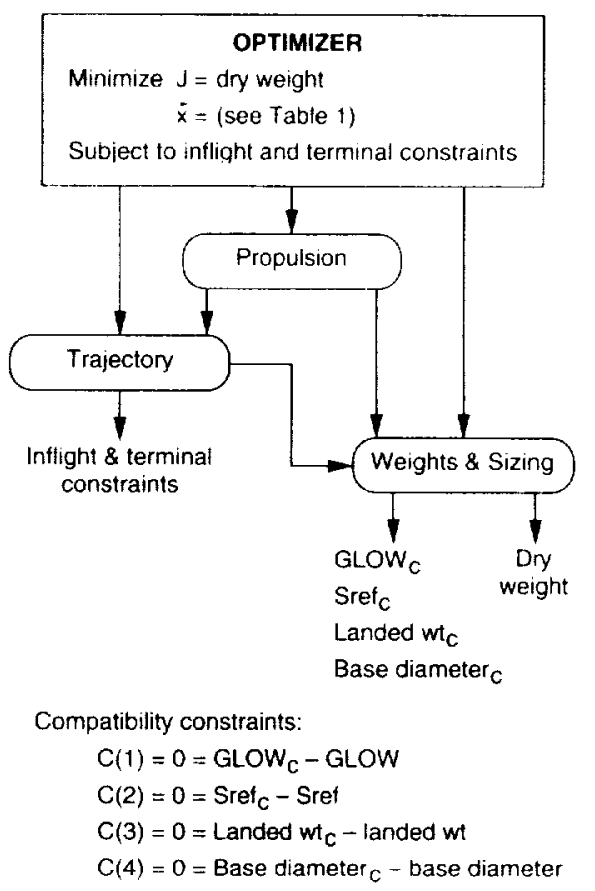

Fig. 10 Strategy for sequential compatibility-constraint solution.

relative increase in dry weight occurs on achieving constraint feasibility. This feasibility is maintained as the dry weight is brought back down to the final value.

In terms of the optimization strategy's overall performance, one may note that a large number of analysis calls was required to achieve each of the solutions listed in Table 2. An analysis call is defined as one pass through the analysis loop, whereas an iteration includes all the calculations required to take a design step (loop convergence at the current design iterate, each point along the line-search, and all tinite-differenced points). Given the fact that this problem was posed with 36 design variables and took approximately 70 iterations to reach the solution, one would expect slightly more than 2590 analysis calls (for 70 objective functions and forward-differenced gradients). However, these solutions required 4-5 times as many analysis calls. The source of this increased computational requirement is that while this strategy may provide the most straightforward approach to integrating an existing set of tools, multidisciplinary feasibility must be established at every point where either the objective function or constraints are evaluated. In fact, this level of compatibility across disciplinary boundaries is only needed at the solution. In addition to the added computational expense, this approach can lead to numerical difficulties when finite-differencing if the analysis loop is not converged tightly. In this investigation, a loop tolerance $(\Sigma$ of Fig. 4) on the order of $1.0 \times 10^{-12}$ was required to produce accurate derivatives. Without such a small tolerance, finite-difference errors led to spurious results. These computational difficulties are overcome in the next solution strategy. 
Table 3 Single-stage-to-orbit launch-vehicle design solution: summary for sequential compatibility-constraint method

\begin{tabular}{|c|c|c|}
\hline & \multicolumn{2}{|c|}{ From initial point } \\
\hline & 1 & 2 \\
\hline \multicolumn{3}{|l|}{ Design variables } \\
\hline GLOW. lb & $2.1675 e+06$ & $2.1668 e+06$ \\
\hline$S_{\mathrm{rcf}}, \mathrm{ft}^{2}$ & $4.0500 e+03$ & $4.0497 \mathrm{e}+03$ \\
\hline Landed weight, lb & $2.2113 \mathrm{e}+05$ & $2.2112 \mathrm{e}+05$ \\
\hline Base diameter, $\mathrm{ft}$ & 27.928 & 27.922 \\
\hline Launch azimuth, deg & 42.328 & 42.328 \\
\hline Vehicle liftoff $T / W$ & 1.2000 & 1.2000 \\
\hline$\% \mathrm{LH}_{2}$, mode 1 & 6.2614 & 6.2596 \\
\hline Mode 2 mixture ratio & 6.9893 & 6.9996 \\
\hline Nozzle area ratio, retracted & 59.541 & 59.801 \\
\hline Nozzle area ratio, extended & 119.08 & 119.60 \\
\hline \multicolumn{3}{|l|}{ Propulsion mode transition } \\
\hline Mach number & 7.3062 & 7.3117 \\
\hline Nozzle transition Mach number & 0.8980 & 0.9090 \\
\hline$F_{Z}$ boundary duration, s & 63.400 & 56.819 \\
\hline Total trajectory time, $s$ & 377.80 & 377.60 \\
\hline \multicolumn{3}{|l|}{ Performance } \\
\hline Final objective (dry weight, lb) & $1.92788 \mathrm{e}+05$ & $1.92785 \mathrm{e}+05$ \\
\hline Number of iterations & 70 & 77 \\
\hline Number of analysis calls & 3162 & 3431 \\
\hline CPU time, $h$ & 4.0 & 4.6 \\
\hline
\end{tabular}

\section{Sequential Compatibility Constraint Method}

In the second approach, the iterative loop of Fig. 4 is replaced by the use of auxiliary variables and compatibility constraints. ${ }^{16-19}$ As shown in Fig. 10, an auxiliary variable and a compatibility constraint are added to the optimization-problem statement for each variable that is required as input to one discipline but is computed by another discipline later in the analysis sequence. Hence, $S_{\text {ref }}$, GLOW, the base diameter, and the landed weight are added as design variables in the Fig. 10 optimizer. In this manner, the iterative loop of Fig. 4 is removed, and configuration control becomes an additional task of the optimizer. By satisfying these four compatibility constraints, a consistent vehicle model is guaranteed. However, as opposed to the approach of Fig. 4, compatibility is required at the solution only. This type of approach may be referred to as "simultaneous analysis and design," since both a consistent and an optimum set of design variables are converged upon simultaneously. ${ }^{20}$

Using this formulation, solutions of the single-stage-to-orbit launch-vehicle design problem were obtained from each of the two starting points listed in Table 1 . These solutions are summarized in Table 3. Once again, although the solution that began at starting point 2 required more iterations, both optimization paths converged to approximately the same point. Note that the solutions obtained through use of the compatibility-constraint approach are approximately $100 \mathrm{lb}$ heavier than those found with the iterative-loop approach. This discrepancy results because the use of larger compatibility tolerances $\left(1.0 \times 10^{-5}\right)$ was possible with this method. When these tolerances were reduced to $1.0 \times 10^{-12}$ (the tolerance level that was required for the iterative-loop solutions), the compatibilityconstraint solutions were within $20 \mathrm{lb}$ of the looped solutions. Recall that in the iterative-loop approach, a compatibility tolerance on the order of $10^{-5}$ was not possible because of the loss of finite-difference accuracy. Hence, a major advantage of the compatibility-constraint approach is that accurate derivatives may be obtained without requiring strict convergence of the disciplinary models. This flexibility may be used to realize reduced computational requirements in preliminary design analyses where coarser approximations are adequate, while maintaining the capability for more accurate solutions as the design progresses. Another computational advantage results from the fact that a consistent vehicle model is only required at the solution. Therefore, disciplinary-model convergence is not required at each design iterate, each point along the line search, and each finite-difference point.

These computational advantages are clear in the number of analysis calls (and corresponding CPU time) required to obtain each solution. Compared to the iterative-loop method, the sequential compatibility-constraint approach requires only $25 \%$ of the computational time to reach approximately the same solution (within $0.05 \%$ in dry weight). Recall that in this approach we have 40 design variables (the original 36 , plus the four auxiliary variables used to establish compatibility). Hence, for 70 iterations, one would expect slightly more than 2870 analysis calls. This estimate matches well with the actual number of analysis calls listed in Table 3 . The only disadvantage to this approach relative to the looped method would be in a situation where solution convergence is not achieved. In that case, one could end up with no valid design information, since model convergence is not guaranteed across disciplinary boundaries. Note that this situation, which could arise in a poorly scaled problem, may be mitigated by adjusting the scaling strategy ${ }^{21}$ or through application of a feasible-point optimization method.

The ascent-trajectory time histories for this case were nearly identical to those obtained with the previous solution strategy (Figs. 5 and 6). Convergence histories are shown for the objective function and norm of the nonlinear constraints beginning from starting point 2 in Figs. 11 and 12. Note the dry-weight scale change in going from Fig. 11 a to Fig. 11b, and the semilog format of Fig. 12. From these figures, it is clear that after approximately five iterations the objective function achieves a near-optimum value, and after approximately 20 iterations the compatibility constraints are satisfied. However, as in the looped approach, it is the convergence of the highly nonlinear trajectory constraints that is causing computational difficulty. Once again, the lack of model smoothness is evident in Fig. 12 and may have hampered the convergence rate. Comparing Figs. 11 and 12 with Figs. 8 and 9, it is clear that the use of compatibility constraints provides another advantage-a smoother design space. That is, by eliminating the loop convergence requirement, a smoother analysis results. This same effect was noted for convergence from starting point 1 .

Note that the approach of Fig. 10 still requires a sequential solution. That is, the propulsion discipline is analyzed first, followed by the trajectory discipline, and then finally the weight and sizing calculations. A sequential solution is required because only the feedback portions of the iterative loop were broken; feedforward

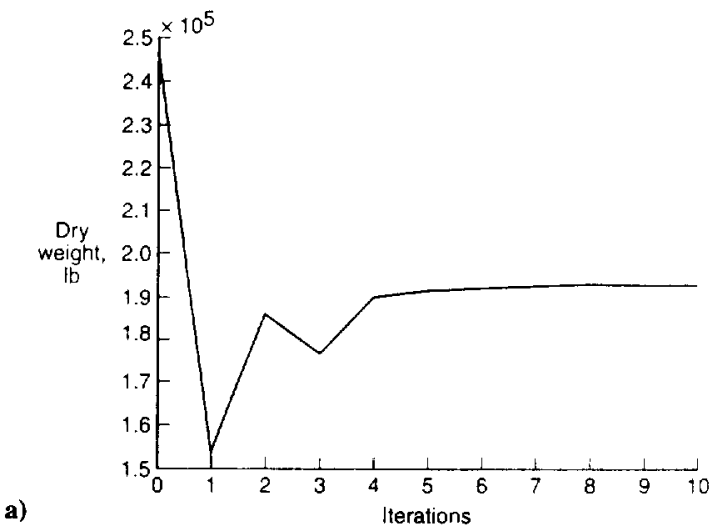

a)

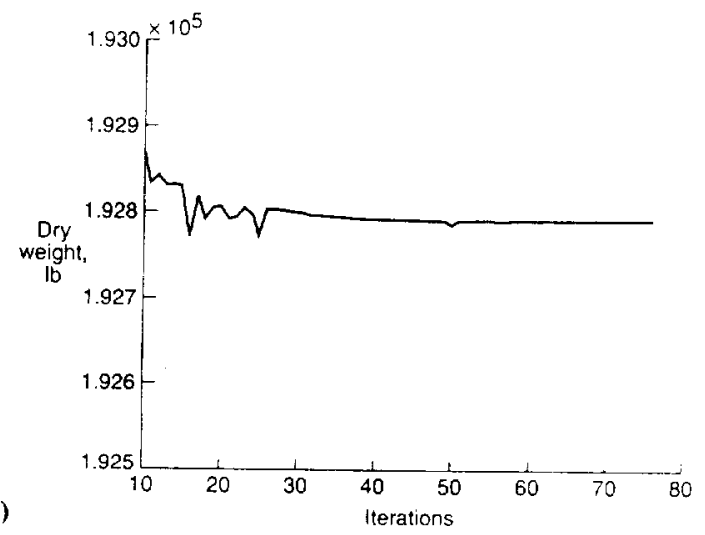

Fig. 11 Objective-function (dry weight) convergence history for the sequential compatibility-constraint solution: a) first 10 iterations and b) final 67 iterations (note scale change). 


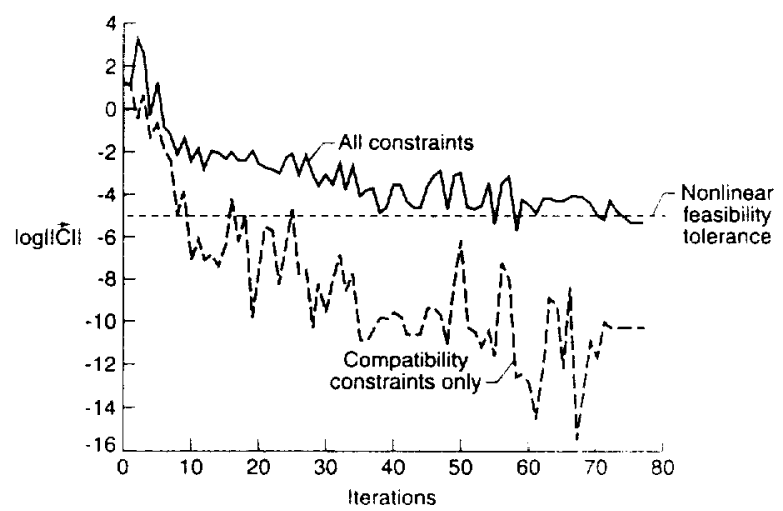

Fig. 12 Nonlinear-constraint convergence history for the sequential compatibility-constraint solution.

from one discipline to the next is still performed (e.g., the mass ratio is computed by the trajectory discipline and used as an input for the weight and sizing analysis). This differs from the approach suggested in Ref. 22, where removal of both the feedforward and feedback communication requirements yields a parallel analysis strategy. A sequential analysis strategy was selected for solution of this problem because it required the addition of only four auxiliary variables and compatibility constraints. In comparison, a parallel strategy would have required an additional 21 variables and constraints, nearly doubling the size of the original problem. Although increasing the problem size does not always result in an increased solution time (the optimization algorithm, the order and sparseness of the design space, and the implementation strategy are all major factors), ${ }^{23.24}$ for a dense, sequential quadratic programming algorithm (such as NPSOL) the solution time is roughiy proportional to the problem size.

\section{Summary}

The present investigation focused on the development of a rapid multidisciplinary analysis and optimization capability for launchvehicle design. In particular, two multidisciplinary optimization strategies were implemented and evaluated for solution of a singlestage-to-orbit launch-vehicle design problem. Weights and sizing, propulsion, and trajectory issues were directly addressed, and the need to maintain a consistent vehicle model across the disciplines was discussed. The approaches were referred to as the iterative-loop method and the sequential compatibility-constraint approach.

Both solution strategies require some disciplinary tool integration and were shown to reach approximately the same solution from two different starting points. Each of these solutions suggests that a dual-fuel, single-stage-to-orbit vehicle with a dry weight of approximately $1.927 \times 10^{5} \mathrm{lb}$, gross liftoff weight of $2.165 \times 10^{6} \mathrm{lb}$, and length of $181 \mathrm{ft}$ is attainable.

Currently, within the launch-vehicle design community, methods of the general form of the iterative-loop approach are predominantly used. In the present investigation, the manner in which disciplinary coupling is treated was shown to have a direct effect on optimization convergence and the computational effort required to reach the solution. This analysis has shown that use of the sequential compatibility-constraint approach has several advantages relative to the iterative-loop approach. These advantages include: 1) being 3-4 times more computationally efficient (for this problem), 2) providing greater flexibility in the way in which consistency is maintained across disciplinary boundaries, and 3) a smoother design space. The only disadvantage of the compatibility-constraint approach is in situations when the optimizer terminates without reaching the solution on account of poor scaling or model nonsmoothness. Because multidisciplinary feasibility is only guaranteed at a solution in this approach, the design information could be invalid. However, the likelihood of optimization termination without constraint satisfaction could be lessened with the use of a more robust scaling strategy or a feasible-point optimization algorithm.

\section{References}

I Wurster, K. E., Rowell, L. F., and Peach, L. L., "The Next Generation Manned Launch System-A Complex Decision." AIAA Paper 93-4160, Sept. 1993.

${ }^{2}$ Freeman, D. C. Wilhite, A. W., and Talay, T. A., "Advanced Manned Launch System Study Status," Paper 91-193, International Astronautical Federation. Oct. 1991.

${ }^{3}$ Wilhite, A. W., Bush, L. B., Cruz, C. I., Lepsch, R. A., Morris, W. D., Stanley, D. O., and Wurster, K. E., "Advanced Technologies for Rocket Single-Stage-to-Orbit Vehicles," Journal of Spacecraft and Rockets, Vol. 28, No. 6, 1991, pp. 646-651.

${ }^{4}$ Eldred, C. H. Powell, R. W., and Stanley, D. O. "Single Stage Rocket Options for Future Launch-Vehicles," AIAA Paper 93-4162, Sept. 1993

${ }^{5}$ Stanley, D. O., Engelund, W. C., Lepsch, R. A., McMillin, M. L., Wurster K. E., Powell, R. W., Guinta, A. A., and Unal, R., "Rocket-Powered SingleStage Vehicle Configuration Selection and Design." Journal of Spacecrafi and Rockets, Vol 31. No. 5, 1994, pp. 792-798; also AlAA Paper 93-1053, Feb. 1993.

"Lepsch, R. A. Stanley, D. O., and Unal, R., "Application of Dual-Fuel Propulsion to a Single Stage AMLS Vehicle," Journal of Spacecraft and Rockets, Vol. 32, No. 3, 1995; also AIAA Paper 93-2275. June 1993.

${ }^{7}$ Engelund, W. C., Stanley, D. O., McMillin, M. L.. and Unal, R., "Aerodynamic Configuration Design Using Response Surface Methodology Analysis," AIAA Paper 93-3967. Aug. 1993.

${ }^{k}$ Limerick C. . private communication, Pratt \& Whitney, West Palm Beach. FL, June 1993

${ }^{9}$ Brauer, G. L., Cornick, D. E., and Stevenson, R., "Capabilities and Applications of the Program to Optimize Simulated Trajectories," NASA CR2770, Feb. 1977

II'Olds, J. R., "System Sensitivity Analysis Applied to the Conceptual Design of a Dual-Fuel Rocket SSTO," AIAA Paper 94-4339, Sept. 1994.

${ }^{11}$ Gill, P. E., Murray, W., Saunders, M. A., and Wright, M. H., "Users Guide for NPSOL (Version 4.0): A Fortran Package for Nonlinear Programming." TR SOL 86-2, Dept. of Operations Research, Stanford Univ.. Jan. 1986.

${ }^{12}$ Eldred, C. H., and Gordon, S. V., "A Rapid Method for Optimization of the Rocket Propulsion System for Single-Stage-to-Orbit Vehicles," NASA TN D-8078, July 1976.

${ }^{13}$ Nguyen, H. N., "FONSIZE: A Trajectory Optimization and Vehicle Sizing Program," AIAA Paper 93-1100, Feb. 1993.

${ }^{14}$ Pribnow, R. S , "Launch Vehicle Synthesis Methodology," SAWE Paper 1992. May 1991.

${ }^{15}$ Cramer, E. J.. Dennis, J. E., Frank, P. D., Lewis, R. M., and Shubin, G. R., "Problem Formulation for Multidisciplinary Optimization," SIAM Journal of Optimization, Vol. 4, No. 4, 1994, pp. 754-776; also AIAA Paper 92-4752, Sept. 1992

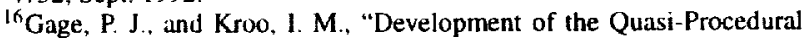
Method for Use in Aircraft Configuration Optimization," AIAA Paper 924693, Sept. 1992.

${ }^{17}$ Braun, R. D., Kroo, I. M., and Gage, P. J., "Post-Optimality Analysis in Aerospace Vehicle Design," AIAA Paper 93-3932, Aug. 1993.

${ }^{18}$ Gabriele, G. A., "Application of the Generalized Reduced Gradient Method to Conceptual Aircraft Design," NASA CP 2327, April 1984.

${ }^{19}$ Betts, J. T., "Frontiers in Engineering Optimization," Journal of Mechanisms, Transmissions, and Automation in Design, Vol. 105, June 1983, pp. $15 t-154$

${ }^{20}$ Balling, R. J., and Sobieszczanski-Sobieski, J., "Optimization of Coupled Systems: A Critical Overview of Approaches," AIAA Paper 94-4330, Sept. 1994.

${ }^{21}$ Hallman, W. "Optimal Scaling Techniques for the Nonlinear Programming Problem," AlAA Paper 94-4417, Sept. 1994.

${ }^{22}$ Kroo, I., Altus, S., Braun, R., Gage, P., and Sobieski, I., "Multidisciplinary Optimization Methods for Aircraft Preliminary Design," AIAA Paper 94-4325, Sept. 1994.

${ }^{23}$ Betts, J. T. Eldersveld, S. K., and Huffman, W. P., "A Performance Comparison of Nonlinear Programming Algorithms for Large Sparse Problems," AIAA Paper 93-3751, Aug. 1993.

${ }^{24}$ Cramer, E. J., Huffman, W. P., and Mastro, R. A., "Sparse Optimization for Aircraft Design," AIAA Paper 93-3935, Aug. 1993.

J. A. Martin Associate Editor 